\title{
Photometry of Some Recent Gamma-ray Bursts
}

\author{
J. Kóbori*, J. Kelemen*, P. Veres ${ }^{\dagger}$, B. Cenko** and D. Fox ${ }^{*}$ \\ *Konkoly Observatory \\ ${ }^{\dagger}$ Eötvös University, Bolyai Military University \\ ${ }^{* *}$ CALTECH \\ ${ }^{\ddagger} P S U$
}

\begin{abstract}
We present the results of the optical, X-ray and gamma-ray analysis of some recent GRBs. The data were obtained by the automated P60 telescope and the Swift telescope (UVOT, XRT and BAT). We present some example fits for the lightcurves. The data reduction and the investigations were made by the Konkoly Observatory HEART group (http://www.konkoly.hu/HEART/index.html).
\end{abstract}

Keywords: gamma-ray bursts, astronomical observations, x-ray

PACS: $98.70 . \mathrm{Rz}$, 95.85.Pw, 98.70.Qy

\section{PHOTOMETRIC DATA REDUCTION}

The UVOT photometry was done using NASA's HEASOFT software package. This package provides a complete assistance for doing photometry for measurements done by various telescopes (e.g. Swift, CGRO, INTEGRAL).

For photometry, contrary to the suggestions of the software manual, we explored various aperture sizes between 1" and 10" (instead of keeping 5" at all times) to get the most usable data. Then we chose those aperture sizes, which provided the most accurate results. For a given filter band we used the best aperture size, meaning that in some cases when obtaining photometry for a given GRB we used various apertures depending on the filter band.

All UVOT magnitudes are in the Standard UVOT Photometric System [2]. Magnitudes obtained by ground based telescopes were taken from GCNs (in detail see at references [7]). When converting magnitudes to fluxes, we used the methods described in $[3,4,5]$. Magnitudes are not corrected for galactic extinction. X-ray data is taken from the XRT observations available from [6]. According to our results we suggest 19 magnitude as acceptable faintest limit in the UVOT photometry system.

We used the mpfit package [1] for fitting broken power-law functions to the lightcurves with the $F_{v} \propto t^{\alpha}$ convention.

\section{INDIVIDUAL AFTERGLOWS}

GRB 080721: The afterglow was detected by several telescopes, but due to the lack of ground based observations we could not fit the data at the late times. However, the UVOT magnitudes show a lightcurve flattening about 6 ks. After this time the UVOT slope in "WHITE" filter is $-0.70 \pm 0.18$, which is inconsistent with the one inferred from filter R, $\alpha_{R} \sim-0.95$ (from 10.6 to 26.19 hours after the trigger), unless there was an additional steepening in the lightcurve. The redshift was inferred from the Lyman- $\alpha$ absorption line and it is $z=2.602$, while other lines (O I, Si II, C II, Si IV, C IV, Fe II and Al II) suggest $z=2.591$.

GRB 081203A: The $\alpha$ index in the R band disagrees with those in the GCNs ( $\alpha_{R, I}=-0.66$ between 6 and $\left.12 \mathrm{ks}\right)$, but after this time the results were similar. The redshift based on the Lyman- $\alpha$ line is about 2.1.

GRB 090102: This is a well studied GRB [see GCN Circular: 8761, 8763, 8764, 8771, 8772, 8773, 8778, 8780]. Its redshift is 1.547. The observations have already begun 43 seconds after the BAT trigger and the TAROT magnitudes even show the brightening interval of the lightcurve. Furthermore, in the UVOT's V and UVW1 band there is an additional rebrightening around $800 \mathrm{~s}$, which can be also seen in the X-ray band, but unfortunately, this part is lacking in ground based observations. The lightcurve has a flattening around $1 \mathrm{ks}$. The redshift of this burst is $z=1.547$.

GRB 090313: The afterglow brightened during the first $1.3 \mathrm{ks}$, then faded until $\sim 10 \mathrm{ks}$. Starting this time the plateau phase appeared, which lasted at least until $\sim 19$ hours after the trigger. Then the average slope for $\mathrm{g}, \mathrm{r}, \mathrm{i}, \mathrm{z}$ 


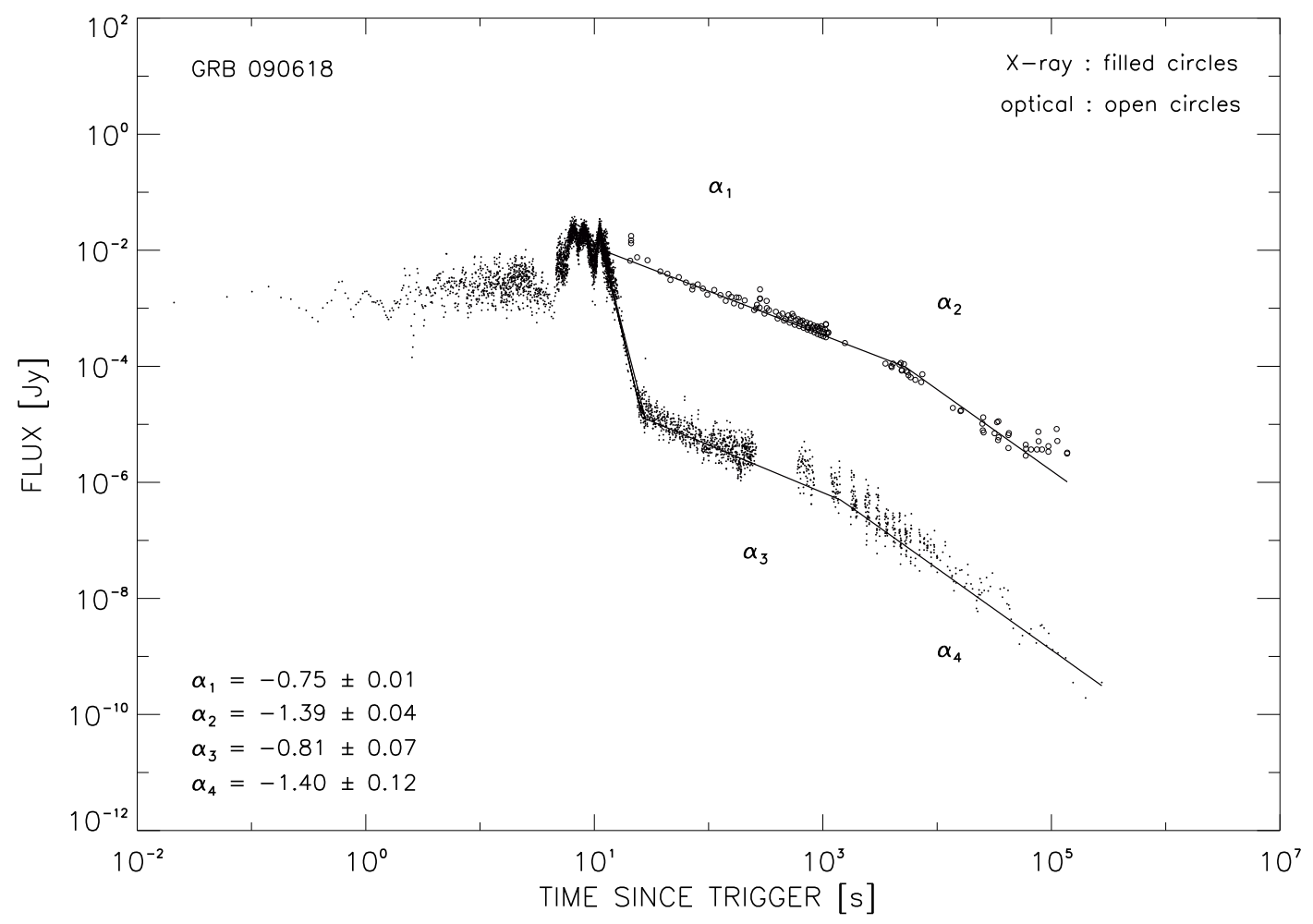

FIGURE 1. An example of the lightcurve fitting. In the case of GRB090618 we determined the break in the lightcurve. The open circles indicate the optical data, which consists the following filters: V, R, I, J, H, K, r', i', z'. On the figure, we fitted all the optical bands together to demonstrate the similar temporal slopes in the optical and the X-ray bands. In the R filter 2 outlier points and BAT measurements were entirely excluded from the fit.

bands changed to $\bar{\alpha}=-1.77$. The redshift is $z=3.375$.

GRB 090618: This burst was very luminous with a bright afterglow. The KAIT observations show an initial decline until $92 \mathrm{~s}$ then a rise at $120 \mathrm{~s}$. After that time the lightcurve showed two breaks: the first occurred around $600 \mathrm{~s}$, the second at 14.6 hours after the trigger. However, in the UVOT observations we found a third break (from $\bar{\alpha}=-0.64$ to $\bar{\alpha}=-1.07$ ), which is in between the previous two, at $\sim 8000 \mathrm{~s}$, but ground based telescopes did not report about such an event. This break explains why after the $(8000 \mathrm{~s})$ epoch the UVOT slopes did not match to the slopes reported in GCNs (between $600 \mathrm{~s}$ and 14.6 hours $\bar{\alpha}=-0.76$ ). From RTT images I. Khamitov et al. determined that 7.68, 8.64 and 9.58 days after the burst the afterglow had a constant $22.3 \pm 0.01$ magnitude. The redshift is $\mathrm{z}=0.54$.

GRB 090812: Measurements from the RAPTOR telescope system shows the lightcurve rising up to $\sim 70 \mathrm{~s}$, which is followed by a steady decay. The redshift is 2.452 .

\section{CONCLUSION}

In this work we aimed to produce photometric data with the highest possible accuracy. Our sample consisted of relatively bright and well observed bursts in order to achieve a reliable data set with our reduction method. We excluded those large error measurements which we had to disregard during our fitting procedure. According to our results we suggest 19 magnitude as the faintest limit when using the UVOT system. 


\section{ACKNOWLEDGMENTS}

Thanks to Bob Wiegand for the help us to make HEASOFT working correctly. This work was partially supported by OTKA grant K077795 (P.V.).

\section{REFERENCES}

1. http://purl.com/net/mpfit

2. T.S. Poole et al. Photometric calibration of the Swift ultraviolet/optical telescope, MNRAS, 383, 627

3. Model atmospheres broad-band colors, bolometric corrections and temperature calibrations for O - M stars, M.S. Bessell , F. Castelli, and B. Plez, Astron. Astrophys. 333, 231-250 (1998)

4. Dark Bursts in the Swift Era: The Palomar 60 inch-Swift Early Optical Afterglow Catalog, S. B. Cenko, J. Kelemen , F. A. Harrison, D. B. Fox , S. R. Kulkarni , M. M. Kasliwal, E. O. Ofek , A. Rau , A. Gal-Yam, D. A. Frail and D.-S. Moon, The Astrophysical Journal, Volume 693, Issue 2, pp. 1484-1493 (2009)

5. The Sloan Digital Sky Survey Photometric System, M.Fukugita, T. Ichikawa, J. E. Gunn, M. Doi and K. Shimasaku, D. P. Schneider, The Astronomical Journal, Volume 111, Number 4

6. Methods and results of an automatic analysis of a complete sample of Swift-XRT observations of GRBs, Evans, P. A., Beardmore, A. P., Page, K. L., et al. MNRAS, 2009, Volume 397, 1177

7. GCN Circulars:

GRB080721: 7998, 7999, 7990

GRB081203A: $\quad 8596,8604,8615,8617,8618,8619,8629,8632,8645,8695$

GRB090102: $\quad 8761,8763,8764,8771,8772,8773,8778,8780$

GRB090313: $\quad 8983,8985,8989,8992,8995,8997,8999,9001,9002,9606,9008$

GRB090618: $\quad 9513,9517,9520,9522,9529,9531,9536,9539,9541,9542,9548,9563,9575,9576,9597,9613$

GRB090812: $\quad 9769,9770,9773,9778,9779$ 
TABLE 1. Alpha indices. $\star$ indicates, that the slope is consistent with other measurements. $\dagger$ indicates a difference in the indices. • means, that it is an average value and is taken from GCNs (in some cases we could not fit the data, e.g. there was only the slope reported). Where two values are presented, the first applies to early times, the second to the late times.

\begin{tabular}{|c|c|c|c|c|c|c|}
\hline & GRB080721 & GRB081203A & GRB090102 & GRB090313 & GRB090618 & GRB090812 \\
\hline V & $-1.09 \pm 0.07^{\star}$ & $-1.54 \pm 0.03$ & - & - & $\begin{array}{c}-0.62 \pm 0.04 \& \\
-1.06 \pm 0.10\end{array}$ & - \\
\hline B & $-1.38 \pm 0.20$ & $-1.45 \pm 0.02$ & - & - & $-0.60 \pm 0.04$ & - \\
\hline $\mathrm{U}$ & $-1.34 \pm 0.23$ & $-1.33 \pm 0.01$ & - & - & $\begin{array}{c}-0.70 \pm 0.01^{\star} \& \\
-1.04 \pm 0.05\end{array}$ & $-0.49 \pm 0.10^{\dagger}$ \\
\hline UVW1 & - & $-1.61 \pm 0.09$ & - & - & $\begin{array}{c}-0.68 \pm 0.03^{\star} \& \\
-1.15 \pm 0.06\end{array}$ & - \\
\hline UVM2 & - & - & - & - & $-0.72 \pm 0.04^{\star}$ & - \\
\hline UVW2 & - & - & - & - & $\begin{array}{c}-0.55 \pm 0.06 \& \\
-1.03 \pm 0.04\end{array}$ & - \\
\hline WHITE & $\begin{array}{c}-1.25 \pm 0.01^{\star} \& \\
-0.70 \pm 0.18\end{array}$ & - & $-1.35 \pm 0.14^{\dagger}$ & - & $\begin{array}{c}-0.73 \pm 0.01 \& \\
-1.07 \pm 0.03\end{array}$ & - \\
\hline $\mathrm{R}$ & $\sim-0.95^{\bullet}$ & $-1.73 \pm 0.01^{\dagger, \star}$ & $\begin{array}{c}-1.60 \pm 0.02^{\bullet} \\
\sim-0.9^{\bullet}\end{array}$ & $-1.61 \pm 0.01$ & $\begin{array}{l}-0.69 \pm 0.01^{\star} \\
-1.27 \pm 0.01^{\star}\end{array}$ & - \\
\hline $\mathrm{I}$ & - & $-1.75 \pm 0.03^{\dagger, \star}$ & - & - & - & \\
\hline $\mathrm{J}$ & - & - & - & $-0.76 \pm 0.01$ & $-0.83 \pm 0.04$ & - \\
\hline $\mathrm{H}$ & - & - & - & - & $-0.86 \pm 0.04$ & - \\
\hline $\mathrm{K}$ & - & - & - & $-0.55 \pm 0.03$ & - & - \\
\hline $\mathrm{g}^{\prime}$ & - & $-2.27 \pm 0.37$ & $\sim-0.9^{\bullet}$ & - & - & $-1.98 \pm 0.12$ \\
\hline r' & - & - & $-1.00 \pm 0.24$ & - & $-0.77 \pm 0.03$ & $-1.24 \pm 0.01$ \\
\hline i' & - & - & $\sim-0.9^{\bullet}$ & - & $-0.73 \pm 0.02$ & $-1.21 \pm 0.02^{\star}$ \\
\hline $\mathrm{z}^{\prime}$ & - & - & - & - & $-0.70 \pm 0.04^{\star}$ & - \\
\hline
\end{tabular}

